PREPARED FOR SUBMISSION TO JINST

The $9^{\text {th }}$ international workshop on Semiconductor Pixel Detectors for Particle AND IMAGING

10-14 DECEMBER, 2018

Academia Sinica, Taipei, Taiwan

\title{
Single Event Upsets in the ATLAS IBL Frontend ASICs
}

\author{
Y. Takubo, ${ }^{a, 2}$ on behalf of the ATLAS collaboration \\ ${ }^{a}$ High Energy Accelerator Research Organization (KEK), 1-1 Oho Tsukuba Ibaraki, 305-0801, Japan \\ E-mail: yosuke.takubo@kek.jp
}

\begin{abstract}
Single event effects like Single Event Upset (SEU) and Single Event Transient (SET) are big concerns in detector operation in high radiation environment. The single event effect are being more visible in the operation of Insertable B-Layer (IBL) located on the $3.3 \mathrm{~cm}$ radius from the beam pipe, as the instantaneous luminosity at the LHC increases. In this paper, studies of single event effects on the front-end ASICs used for the IBL will be described.
\end{abstract}

Keywords: Particle tracking detectors (Solid-state detectors), Front-end electronics for detector readout, Radiation-hard electronics

${ }^{1}$ Copyright 2019 CERN for the benefit of the ATLAS Collaboration . Reproduction of this article or parts of it is allowed as specified in the CC-BY-4.0 license.

${ }^{2}$ Corresponding author. 


\section{Contents}

1 Introduction 1

2 Single Event Upset and Single Event Transient 1

3 Insertable B-Layer 2

$4 \quad$ FE-I4B front-end chip $\quad 3$

5 Measurement of single event effects in FE-I4B 4

5.1 Single event effects in Global memory 4

$\begin{array}{ll}5.2 & \text { Single event effects in Pixel memory }\end{array}$

$\begin{array}{llr}6 & \text { Conclusions } & 8\end{array}$

\section{Introduction}

ATLAS [1] general purpose detector is used for the study of proton-proton ( $p p)$ and heavy-ion collisions at the CERN Large Hadron Collider (LHC). After the long shut-down period over 2013 and 2014 (LS1), ATLAS experiment started data-taking of its $p p$ beam colliding energy of $13 \mathrm{TeV}$ in May 2015 for Run 2. LHC continuously increased an instantaneous luminosity, and it reached more than $2 \times 10^{34} \mathrm{~cm}^{-2} \mathrm{~s}^{-1}$ in stable beam conditions after 2017. Such luminosity is two times larger than LHC design value. The ATLAS experiment collected data of an integrated luminosity of $149 \mathrm{fb}^{-1}$ by the end of Run 2 on December 2018.

Single event effects like Single Event Upset (SEU) and Single Event Transient (SET) are big concerns to operate the silicon detectors in high radiation environment. Since the pixel detector is placed at the innermost region in ATLAS experiment, the single event effects on the front-end chips may become a serious issue, especially for the innermost pixel layer, Insertable B-Layer (IBL) [2]. Due to this close position with respect to the beam collision point, IBL suffers from the largest radiation dose among all the sub-detectors in ATLAS. As LHC increased its instantaneous luminosity, the single event effects were being more visible in the operation of IBL, especially after 2017.

In this paper, measurement results of single event effects in a front-end chip of IBL and their mitigation will be described.

\section{Single Event Upset and Single Event Transient}

There are two types of upset mechanism in single event effect. An ionizing particle can create a bunch of electron-hole pairs along its trajectory by interaction with material. If the particle passes 
through a circuit of latch or shift register, the state of the circuit may be upset. This effect is called as SEU (Fig. 1 (left)). If SEU happens in a configuration memory of the front-end ASIC, the chip looses the configuration parameters and does not work with the correct setting.

On the other hand, in the case that an ionizing particle penetrates a combinational logic, a glitch may be created and propagated to LOAD line of a memory. Then, if the glitch has enough amplitude and duration in coincidence with a clock, the state of memory is changed. This is called as SET (Fig. 1 (right)).
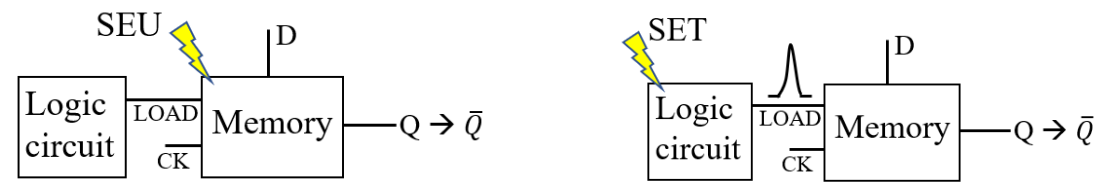

Figure 1. Schematic image of SEU (left) and SET (right).

\section{Insertable B-Layer}

Pixel Detector existing since Run 1 was designed to work at up to an instantaneous luminosity of $1 \times 10^{34} \mathrm{~cm}^{-2} \mathrm{~s}^{-1}$. The instantaneous luminosity of the LHC is expected to increase up to $3 \times 10^{34}$ $\mathrm{cm}^{-2} \mathrm{~s}^{-1}$ after Long Shutdown 2 (LS2) which is planned in $2019-2020$. To maintain or improve the robustness and performance of the ATLAS tracking system given the higher instantaneous and integrated luminosity, a new pixel sensor layer, IBL [2], was installed into the innermost region at the radius of $3.3 \mathrm{~cm}$ from the beam axis in 2014. This was the first upgrade project in ATLAS [1].

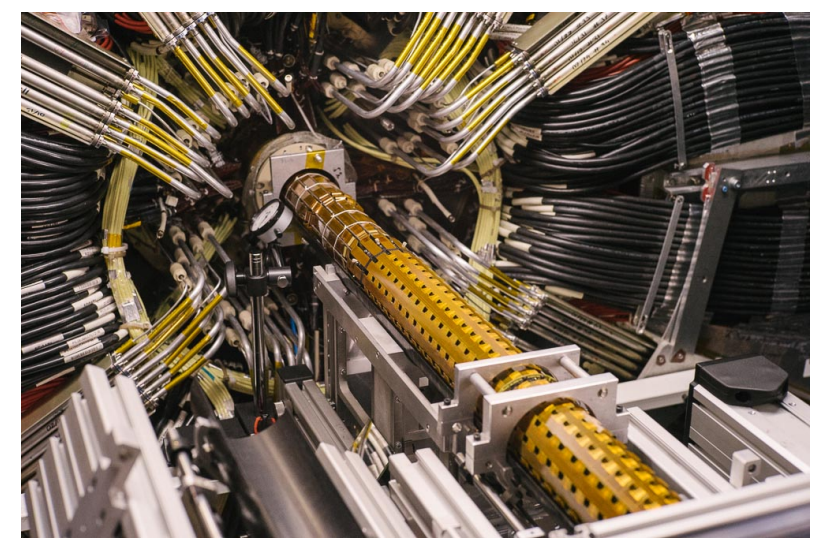

Figure 2. Picture of IBL insertion into ATLAS.

In IBL, two different sensor technologies are adopted: $\mathrm{n}^{+}$-in-n planar sensors [3] and 3D sensors [4]. The pixel size of the sensors is $50 \times 250 \mu \mathrm{m}^{2}$ which is $60 \%$ of the pixel size used for the Pixel Detector $\left(50 \times 400 \mu \mathrm{m}^{2}\right)$. IBL has about 12 million pixels in total. The thickness of the planar and 3D sensors is $200 \mu \mathrm{m}$ and $230 \mu \mathrm{m}$, respectively. An IBL module consists of a sensor bump-bonded to a font-end ASIC (FE-I4B chip [5]) with flex cables which provide electric lines. The modules are mounted on the staves where planar sensors are used for $75 \%$ of the middle 
region in $\eta$ and 3D sensors used in the remaining 25\% (high $\eta$ ). The full IBL consists of 14 staves integrated into a support tube. IBL was transported to ATLAS experimental hall $90 \mathrm{~m}$ underground on May 5, 2014 and installed into ATLAS detector on May 7 as shown in Fig. 2.

After LS1, ATLAS experiment started data-taking in May 2015 for Run 2. IBL has been successfully operated and showed excellent performance even in the condition of more than two times larger instantaneous luminosity of the LHC design value $\left(1 \times 10^{34} \mathrm{~cm}^{-2} \mathrm{~s}^{-1}\right)$. Providing additional hit information at the closest position to the beam collision point, IBL significantly improved the performance of tracking [6] and accordingly $b$-jet tagging [7].

\section{FE-I4B front-end chip}

The FE-I4B front-end chip [5] was developed for IBL with $130 \mathrm{~nm}$ CMOS technology. The chip size is $2 \times 1.8 \mathrm{~cm}^{2}$, about four times larger than FE-I3 chip used for Pixel Detector existing since Run 1. The chip contains read-out circuitry for 26,880 hybrid pixels arranged in 80 columns of $250 \mu \mathrm{m}$ pitch by 336 rows of $50 \mu \mathrm{m}$ pitch. Each pixel contains a free running clock-based amplification stage with adjustable shaping, followed by a discriminator with an independently adjustable threshold. The chip keeps track of the time stamp for each discriminator as well as the 4-bit Time over Threshold (ToT). Information from all firing discriminators is kept in the chip for a latency interval programmable up to $255 \mathrm{LHC}$ clock cycles of $25 \mathrm{~ns}$, and it retrieved if a trigger is supplied within this latency.
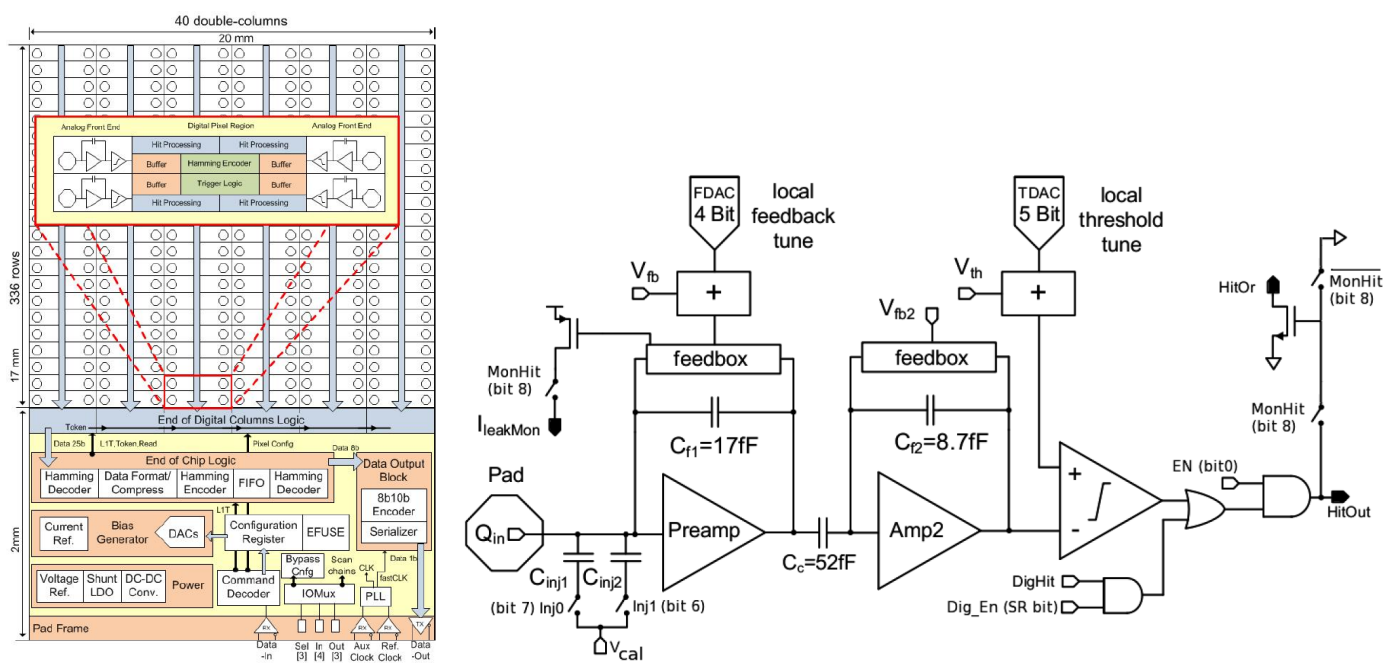

Figure 3. FE-I4 chip diagram (left) and analog pixel schematic diagram (right) [5]. PCM consists of EN, TDAC, $\mathrm{C}_{\text {mathrminj } 1}, \mathrm{C}_{\text {mathrminj2 }}$, MonHit, FDAC and Dig_En (SR bit) in the right figure, where EN enables/disables a pixel, TDAC adjusts threshold, $\mathrm{C}_{\mathrm{inj} 1}$ and $\mathrm{C}_{\mathrm{inj} 2}$ are used to switch on/off an injection capacitors, MonHit enables/disables output for leakage current and self-trigger, FDAC tunes ToT value and Dig_En (SR bit) enables digital injection.

The FE-I4B has many configurable settings to adjust operation parameters that are stored in memories with radiation hardness to SEU. The Global Configuration Memory (GCM) is a RAM block of 32 words of 16 bits each (512 bits in total), that is place in the periphery region in the 
chip (Fig. 3 (left)). GCM is used for setting of the chip level like threshold and ToT. The main requirement of GCM is the high level of tolerance to the SEU. For this reason, FE-I4 uses custom latches with a Dual Interlocked Cell (DICE) architecture, and then triplicate these latches with simple majority logic.

The DICE latch structure is shown in Fig. 4 (left) and consists of cross-coupled inverters. The 4 nodes $\left(n_{1}\right.$ to $\left.n_{4}\right)$ store data as 2 pairs of complementary values. For example, when the stored bit is 0 then $n_{1}, n_{2}, n_{3}, n_{4}=0101$. Even if the state in one of four nodes are flipped, the perturbation is removed by cross-connection between the nodes and gates. However, if two sensitive nodes of the cell storing the same logic state $\left(\mathrm{n}_{1}\right.$ and $\left.\mathrm{n}_{3}\right)$ or $\left(\mathrm{n}_{2}\right.$ and $\left.\mathrm{n}_{4}\right)$ change state due to the effect of a signal particle impact, the immunity is lost and the DICE latch can be upset. For this reason, special layout rules are followed to physically separate sensitive nodes. An enclosed layout nMOS transistor is used to achieve a wide gate with reduced diffusion area, which reduces the upset probability.

GCM is further protected from SEU by a triple redundant structure with three DICE memories. Figure 4 (right) shows a majority logic voting circuit which is implemented in each cell to determine the effective output data. This data bit is corrupted only when more than one latch is upset. Each DICE cell has its own load signal to avoid corrupting the three latches if a transient upset occurs in the preceding combinatorial logic.
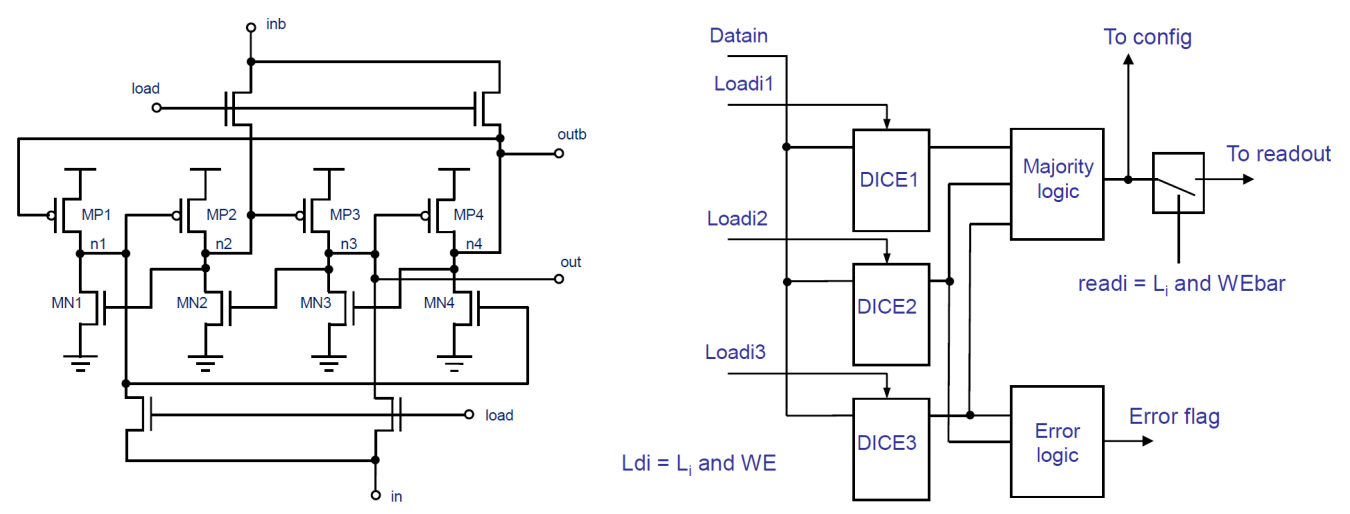

Figure 4. Single DICE latch structure (left) and triple redundant structure for storing each bit (right) [5].

In addition to GCM, each pixel has 13-bit memory, so called Pixel Configuration Memory (PCM) for the pixel level setting of ToT, threshold, enabling or disabling the pixel, etc. as shown in Fig. 3 (right). The PCM uses the custom latches with a DICE architecture.

\section{Measurement of single event effects in FE-I4B}

The single event effects started to be visible in IBL since 2017 as integrated luminosity of $p p$ beam collision increased at LHC. The bit flip rate in GCM and PCM was measured to understand the single event effect quantitatively. In this section, the measurement results are described.

\subsection{Single event effects in Global memory}

A FE-I4B chip has a functionality to readout configuration stored in GCM. The effect of a single event effect in GCM was studied by comparing the initial configuration values and those in a 
data-taking with $p p$ collisions.

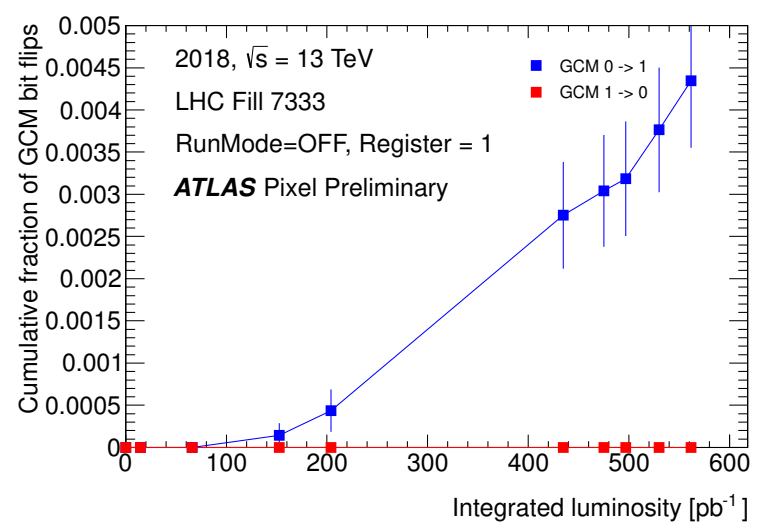

Figure 5. Cumulative fraction of bit flips by the single event effects in GCM of FE-I4B as a function of an integrated luminosity [8].

Figure 5 shows a cumulative fraction of bit flips in GCM as a function of an integrated luminosity. The bit flip rate from 0 to 1 is much higher than the opposite. This suggests that the bit flip is cased by SET (glitches) on a LOAD line in the memory, setting the input register value as 1 , where the register values are refreshed several times during LHC fill. No real SEU transition from 1 to 0 is observed during the fill, thanks to the triple redundant memory design.

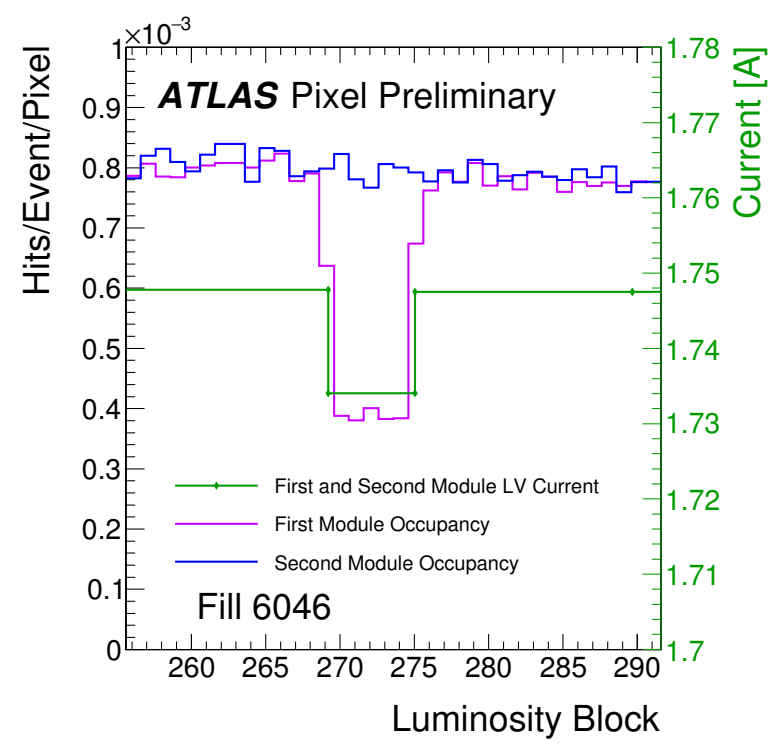

Figure 6. The Low Voltage (LV) current consumption (green) and hits per event per pixel for two IBL modules are plotted as a function of LB. One LV service lines provides current for two modules which are plotted separately. During the data taking, at LB 268, a drop in the LV current consumption can be observed. At the same time, a drop in occupancy is observed in one of the two DAQ modules that share the same LV supply. At LB 277, the critical DAQ module was manually reconfigured bringing back the LV current and the hit occupancy to the values before the SEU [9]. 
Figure 6 shows the Low Voltage (LV) current consumption (green) and hits per event per pixel for two IBL modules as a function of Luminosity Block (LB), where one LB corresponds to about 1 minute. The occupancy of the module impacted by the single event effect is halved. In addition, the LV current changes as one of the two FEs within the module and is no longer configured correctly. Special software called Quick Status (QS) was used in the IBL DAQ system to detect modules that are not working correctly and perform reconfiguration of the chips on those modules. When the corrective action is activated the occupancy and original LV current consumption are restored.

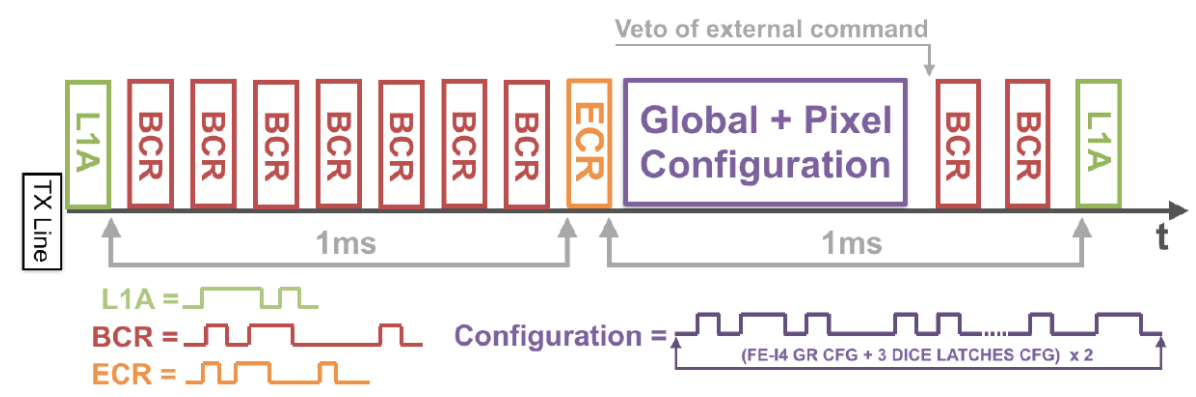

Figure 7. Reconfiguration mechanism at ECR timing for reconfiguration of GCM and PCM.

The mechanism to reconfigure GCM was developed to mitigate the single event effects. The Event Counter Reset (ECR) signal is provided to all the sub-detectors in ATLAS every 5 seconds from the ATLAS Level-1 Central Trigger Processor (CTP) in order to maintain the event synchronization, and there is $1 \mathrm{~ms}$ dead time holding a Level-1 trigger before and after ECR signal as shown in Fig. 7. The Data AQuisition (DAQ) system for IBL was modified to reflesh GCM during $1 \mathrm{~ms}$ time window after ECR. With this new mechanism, the modules affected by the single event effects can be recovered without creating additional dead time in ATLAS data-taking since 2017.

\subsection{Single event effects in Pixel memory}

As like GCM, the configuration stored in PCM also can be readout. The initial configuration values are compared with those after certain period of data-taking to see the single event effects.

Figure 8 shows an average rate of bit flips in PCM per $\mathrm{fb}^{-1}$ as a function of bit number $(0-12)$. On the left figure, the shift register was set to 1 , and $0 \rightarrow 1$ flips dominate. This indicates effect of SET (glitches) on the LOAD line of the memory, while low rate of $1 \rightarrow 0$ flips is considered due to real memory SEU. On the right figure, the shift register is set to 0 and $1 \rightarrow 0$ flips dominate. The values of the shift register are refreshed several times during LHC fill to keep the same value in the shift register. There are factor of two more frequent flips in the bit-8, probably due to specific layout of that memory bit.

The mechanism to reconfigure PCM was developed to mitigate the single event effects. As done for reconfiguration of GCM, $1 \mathrm{~ms}$ of dead time after ECR signal is used to reconfigure PCM. Since $1 \mathrm{~ms}$ is not enough to reconfigure all PCM in a FE-I4 chip, three latches out of 13 in one pixel double column are reconfigured every 5 seconds of ECR timing, where FE-I4B hosts 40 double columns in total. In this condition, the same pixel is reconfigured every 11 minutes. This action does not create additional dead time in the ATLAS data-taking. 

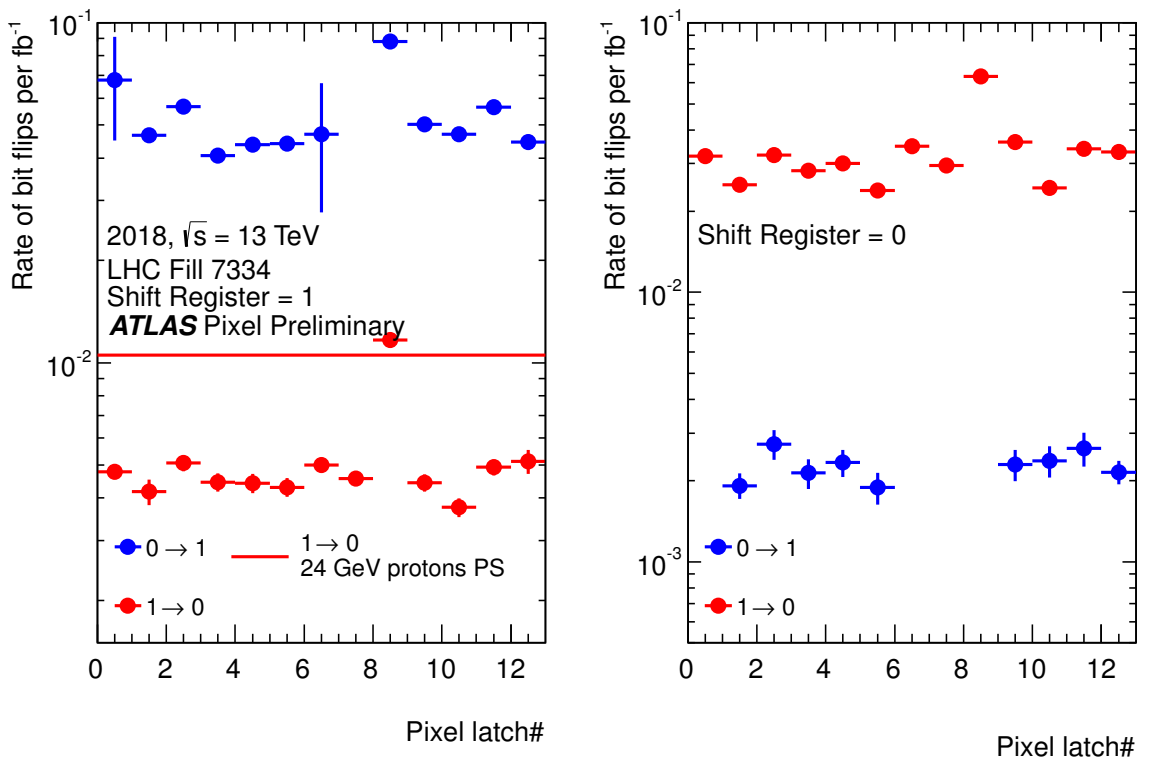

Figure 8. Average rate of bit flips in PCM per $\mathrm{fb}^{-1}$ as a function of bit number $(0-12)$. The Shift Register was set to " 1 " on the left figure and " 0 " on the right figure. The extrapolation of the measurement of SEU rate with $24 \mathrm{GeV}$ protons on CERN PS is shown with a red line on the left plot. During CERN PS measurement the value of SR was not refreshed which may explain higher rate of bit flips due to SET contribution. There are different chips on slightly different locations on two figures, so part of the difference between two figures may come from the chips to chip process variations, tuning and particle flow differences [8].

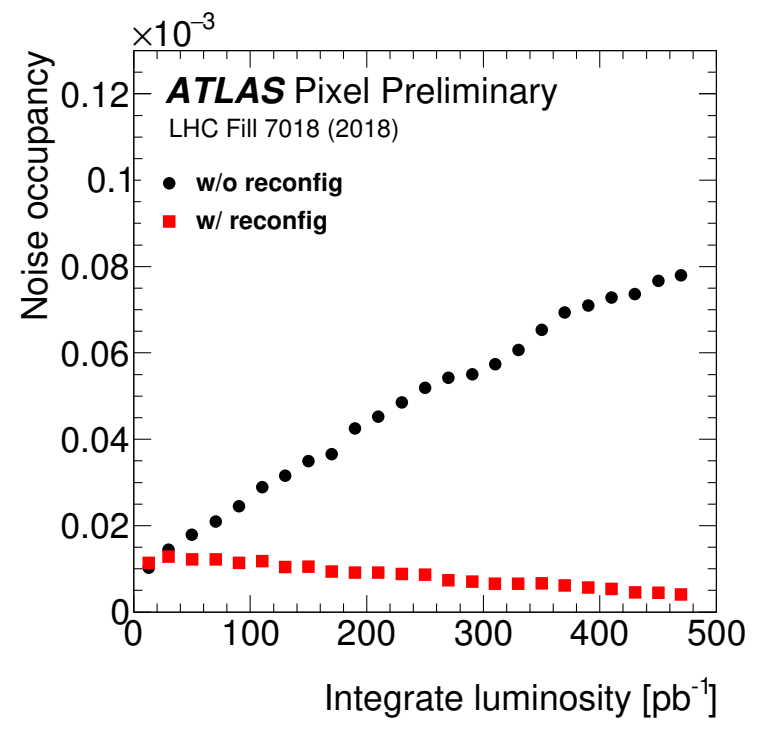

Figure 9. Noise occupancy in IBL 3D modules as a function of integrated luminosity with and without mechanism of pixel register reconfiguration at ECR timing [10].

The mechanism of PCM reconfiguration was tested with IBL during data-taking in 2018. Figure 9 shows the noise occupancy in IBL 3D modules as a function of integrated luminosity with 
and without mechanism of PCM reconfiguration. Without the mechanism, the single event effects cause an increase of the noise occupancy during the run. On the other hand, the noise decreases as a function of integrated luminosity with the mechanism because the reconfiguration is performed every 11 minutes for the same PCM but time to take the same amount of an integrated luminosity becomes longer during a run due to decrease of an instantaneous luminosity.

\section{Conclusions}

Single event effects on FE-I4B front-end chip are being visible in IBL especially after 2017. The bit flip rate in GCM and PCM was measured to understand the single event effect quantitatively. The measurement results indicate that SEU is well suppressed by a triple redundant structure with DICE latches for GCM and a DICE architecture for PCM. In addition, the effect of SET is much larger than that of SEU for FE-I4 chips in IBL.

Mechanism to reconfigure GCM and PCM was developed to mitigate the single event effects by using an ECR signal without adding extra-dead time in ATLAS data-taking. The reconfiguration mechanism could reduce the single event effects to a negligible level in the operation of a FE-I4 chip.

\section{References}

[1] ATLAS Collaboration, The ATLAS Experiment at the CERN Large Hadron Collider, JINST 3 S08003, 2008.

[2] The ATLAS IBL collaboration, Production and integration of the ATLAS Insertable B-Layer, JINST 13 (2018) T05008.

[3] C. Goessling et al., Planar $n^{+}-$in-n silicon pixel sensors for the ATLAS IBL upgrade, Nucl. Instrum. Meth. A 650 (2011), 198-201.

[4] Cinzia Da Via et al., 3D silicon sensors: Design, large area production and quality assurance for the ATLAS IBL pixel detector upgrade, Nucl. Instrum. Meth. A 694 (2012), 321-330.

[5] FE-I4 Collaboration, The FE-I4B Integrated Circuit Guide.

[6] The ATLAS Collaboration, Public Tracking Performance plots, IDTR-2015-007, https://atlas.web.cern.ch/Atlas/GROUPS/PHYSICS/PLOTS/IDTR-2015-007/.

[7] The ATLAS Collaboration, $b$-tagging performance for early $13 \mathrm{TeV}$ analyses, ATLAS NOTE, ATL-PHYS-PUB-2015-022, https://cds.cern.ch/record/2037697/files/ATL-PHYS-PUB-2015-022.pdf.

[8] The ATLAS Collaboration, Public Pixel Tracker Plots for Collision Data, PIX-2018-007, https://atlas.web.cern.ch/Atlas/GROUPS/PHYSICS/PLOTS/PIX-2018-007/.

[9] The ATLAS Collaboration, Public Pixel Tracker Plots for Collision Data, PIX-2017-006, https://atlas.web.cern.ch/Atlas/GROUPS/PHYSICS/PLOTS/PIX-2017-006/.

[10] The ATLAS Collaboration, Public Pixel Tracker Plots for Collision Data, PIX-2018-010, https://atlas.web.cern.ch/Atlas/GROUPS/PHYSICS/PLOTS/PIX-2018-010/. 